\title{
Analysis of Wear Particles Formed in Boundary-Lubricated Sliding Contacts
}

\author{
Aydar Akchurin ${ }^{1,2} \cdot$ Rob Bosman ${ }^{2} \cdot$ Piet M. Lugt $^{2,3} \cdot$ Mark van Drogen $^{3}$
}

Received: 29 February 2016/ Accepted: 27 May 2016/Published online: 16 June 2016

(c) The Author(s) 2016. This article is published with open access at Springerlink.com

\begin{abstract}
The wear process in a sliding contact results in generation of wear debris, which affects the system life. The impact depends on the wear particle properties, such as size, shape and number. In this paper, the wear particles formed during a cylinder-on-disk wear test were examined. PAO additive-free oil, steel-brass and steel-steel contact pairs were employed. A particle isolation procedure was applied, and SEM/EDS analysis was used to validate it. DLS measurements indicated the wear particles radius to be in the range from 230 to $260 \mathrm{~nm}$ for both materials under applied nominal pressures from 87 to $175 \mathrm{MPa}$. AFM data revealed the wear particles size to be in the range from 133 to $175 \mathrm{~nm}$. A slight increase in particles size with load was observed by AFM for both materials and confirmed by DLS for steel samples. AFM measurements were taken to determine thickness, length and width distributions of the wear particles. The number of wear particles per sliding distance and per unit load was estimated to be in the range from 150 to $750 \frac{\mathrm{particles}}{\mathrm{mmN}}$ for steel and approximately $1600-1900 \frac{\text { particles }}{\mathrm{mmN}}$ for brass.
\end{abstract}

Keywords Steel and brass wear particles - Dynamic light scattering - Atomic force microscopy

Aydar Akchurin

a.akchurin@utwente.nl

1 Materials Innovation Institute (M2i), P.O. Box 5008, 2600, GA, Delft, The Netherlands

2 Laboratory for Surface Technology and Tribology, Department of Engineering Technology, University of Twente, P.O. Box 217, 7500, AE, Enschede, The Netherlands

3 SKF Engineering and Research Centre, Kelvinbaan 16, 3439, MT, Nieuwegein, The Netherlands

\section{Introduction}

The wear process results in generation of debris, or particles, of various sizes, shapes, color distributions and chemical compositions [1-3]. The properties of the particles may depend on normal load, sliding speed, environment and many other parameters [4]. The types of wear particles include plate-shaped particles with aspect ratios of $2-10$, produced as a result of accumulated plastic deformation, and ribbon-shaped particles with aspect ratios higher than 10 , produced by abrasion and even spherical particles [1].

Wear particles produce mechanical damage, however, sometimes accompanying effects become even more important. For example, in artificial joint replacements, wear debris triggers the autoimmune reaction and leads to a failure of the joint in a long term [5]. The severity of the reaction is known to be dependent on the wear particles number, size, shape, surface area $[6,7]$. The influence of the wear particles formed in dry sliding was shown to be significant on the transition from the severe "running-in" mode to the mild wear regime $[8,9]$. In case of greaselubricated bearings, the oxidation of the grease thickener and base oil is accelerated in the presence of the wear debris as reported by Hurley et al. [10]. In some situations, the thickener structure can be destroyed by the particles leading to the failure of lubrication and of the bearing [11]. There is also an increasing concern regarding the environmental effect of the metallic particles [12, 13]. Additionally, it is believed that the wear debris also reflects the wear mechanism [14-16], and therefore, significant efforts were devoted to wear particles analysis.

Kato et al. [17] found that, in the case of dry wear and with equipment that could only measure particles larger than $200 \mathrm{~nm}$, all of the considered cases showed the 
maximum number of wear particles of this diameter. This fact indicates that most likely, the actual size of the particles is even smaller. In a series of experimental investigations, Mishina and Hase studied the formation of initial or elementary wear particle in situ using a frictional microscope [18] during the deformation in sliding contacts and examined the surfaces after wear using an atomic force microscope (AFM). Both lubricated and dry contacts were studied to investigate the mechanism of wear particle formation $[19,20]$. The size distribution of the wear particles formed in dry and lubricated sliding contacts of various metals with iron was also obtained [21]. The size of the particles was found to be in the range of tens of nanometers. They concluded that the particle size was determined by the material properties and not by the load and lubrication conditions. However, the number of particles did strongly depend on the load and lubrication conditions. Scherge et al. [22] analyzed wear particles formed in lubricated conditions in chromium-plated steel and gray cast iron pair. A fully formulated oil was used as a lubricant. Using transmission electron microscope (TEM) and automated particle analyzing software, it was found that the size of the particles was around $250 \mathrm{~nm}$ in lateral dimensions. The thickness was found to be not more than $30 \mathrm{~nm}$. Wasche et al. [23] also analyzed wear particles formed in reciprocating lubricated steel-steel contact. As the lubricant, fully formulated and base oil were used. The wear particles were analyzed using scanning electron microscope (SEM). It was concluded that the primary wear particles were of the size of around 5-30 nm and most of the larger particles were agglomerates. It was suggested that the particles were milled down to the small sizes during the reciprocate motion. It was also concluded that although the chemical composition of the wear particles was different (in fully formulated oil, the additives constituents were prevailing, whereas for the base oil, iron was prevailing), their size was approximately the same. Olofsson et al. [12, 24, 25] analyzed airborne wear particles, formed in unlubricated steel-steel sliding contact at several normal loads. The mean particle size was found to be in the range from 200 to $400 \mathrm{~nm}$, with mean values around $350 \mathrm{~nm}$. The distributions, however, were multimodal and in most cases had three distinct peaks. An increase in the total number of particles with load was also reported.

A number of techniques can be employed to analyze the wear particles [26], which can be classified into imaging and non-imaging techniques. The imaging methods include optical, electron (SEM, TEM) and AFM microscopy among others. These tools are potentially capable of measuring shape, size and concentration (also texture) of the particles. However, only a limited amount of particles can be analyzed using imaging procedures and imaging of a significant number of sample spots is required in order to gather statistically reliable information. This makes the imaging methods slow but also subjective. In contrast, the non-imaging techniques, such as dynamic light scattering (DLS), are fast and can analyze a large number of particles simultaneously. The DLS method analyzes the light scattered by particles undergoing a Brownian motion in a suspension. Since the particles are in motion, the scattered light fluctuates, and for the larger particles, timescale of fluctuations is larger than for the smaller ones, which makes it possible to calculate the diameters of the particles [27]. The information about shape, concentration and texture is not available in this case. Therefore, sometimes various techniques are combined.

Particle analysis techniques require sample preparation, and the key issue of most characterization methods is the collection of a sufficient number of particles and/or separation of particles after testing. Common collection techniques include centrifugation and sonication. Centrifugation is necessary to collect most of the particles from the lubricant and possibly to eliminate unwanted contaminants. On the other hand, several researchers showed that the small particles tend to make agglomerations which can be misleadingly considered as large single particles $[18,28,29]$. In order to break these agglomerates, sonication needs to be employed [30, 31].

In the current paper, wear particles formed in boundarylubricated steel on steel and steel on brass sliding contacts were studied. The particles were collected after the cylinder-on-disk test from the oil using an isolation protocol which includes centrifugation and sonication. Polyalphaolefin oil (PAO) additive-free oil was used as a lubricant to minimize chemical effects in the tribo-system. The test conditions were varied to explore the dependence of the particles size on load and materials combination. The particles were analyzed using DLS, SEM (coupled with electron diffraction X-ray spectroscopy, EDS) and AFM techniques. The results will be used for validation of the wear particles size prediction model, which is developed in accompanying paper.

\section{Materials and Methods}

\subsection{Cylinder-on-Disk Wear Tests}

Sliding wear tests were conducted under boundary lubrication conditions with PAO as a lubricant. The viscosity of the oil was $68 \mathrm{cSt}$ at $40{ }^{\circ} \mathrm{C}$. In each test, $4 \mathrm{ml}$ of oil was placed into the disk holder. AISI 52100 steel cylinders with surface roughness (root-mean-square, $R_{q}$ ) of around $50 \mathrm{~nm}$ were worn in pair with bearing cage steel material disks with a roughness of around $610 \mathrm{~nm}$ or with brass disks with 
approximately $760 \mathrm{~nm}$ roughness. The oil, disks and cylinders were supplied by SKF Research Centre, Nieuwegein, the Netherlands. The hardness of the steel disks was $110 \pm 4 \mathrm{HV}$ and that of brass was $112 \pm 2 \mathrm{HV}$. Young's modulus was $88 \mathrm{GPa}$ for brass and $210 \mathrm{GPa}$ for steel disks. The radius of the cylinder was $2 \mathrm{~mm}$, and the width was $6 \mathrm{~mm}$. The normal load was taken to be $2.5,5$ or $10 \mathrm{~N}$ under a constant temperature of $80{ }^{\circ} \mathrm{C}$ and sliding velocity of $0.01 \mathrm{~m} / \mathrm{s}$. Additionally, for the steel disks the temperature was varied. Under $10 \mathrm{~N}$ load, the temperatures used were: 50,80 and $130{ }^{\circ} \mathrm{C}$. No noticeable variation of the wear particles size due to a temperature change in this range was found, since the material properties of steel responsible for wear do not change significantly. Material properties of brass also do not vary much in the considered range of temperatures, and the particles size dependence on temperature was assumed to be negligible and not measured.

Wear tests were performed for 1740 rotations on $13-\mathrm{mm}$ wear track $(4 \mathrm{~h})$. The lubricant with wear particles was collected after the test and stored for further analysis.

\subsection{Surface Roughness and Wear Volume Measurement}

Before the wear test, each disk was indented ( 7 indents) and the initial surface roughness was measured. The indents were placed along the line perpendicular to the wear track. After the wear test, the surface profiles of the worn disks were measured and matched with initial profiles using the indents as markers. The wear volume was then calculated using:

$V=\Delta h \times 2 \pi R \times d$,

where $V$ is the wear volume, $\Delta h$ is the difference between unworn and worn height profiles, $d$ and $R$ are the width and radius of the wear track. Due to the choice of materials, e.g., soft versus hard, the wear is concentrated almost solely on the disk. The wear volume for the cylinder was three orders of magnitude less than the wear volume of the steel disk.

The measurement of roughness was taken using a Keyence VK9700 laser scanning microscope (Keyence, Itasca, IL, USA). Indentations were performed using a LECO microhardness tester LM100AT (LECO, St. Joseph, MI, USA).

\subsection{Particle Isolation Procedure}

In order to prepare wear particles samples for further analysis, the following isolation procedure was applied. First, particles were collected with the oil after the wear test. Isopropanol (2-propanol, IPA) was added to the oil holder which was ultrasonically cleaned for $15 \mathrm{~min}$ at $40{ }^{\circ} \mathrm{C}$. From the experiments, it was found that the cleaning helps to collect a sufficient number of particles in order to perform DLS measurements. If ultrasonic cleaning was not used, sometimes the concentration of particles was not sufficient. IPA was used as a cleaning agent. The mixture of IPA and oil with particles was placed into 40-ml centrifuge tube and centrifuged for $15 \mathrm{~h}$. The upper $30 \mathrm{ml}$ was then discarded, and the tube was filled with deionized water (DI) and centrifuged again for $15 \mathrm{~h}$. During the centrifugation, high-density wear particles (mostly consisting of iron or brass, $\rho \approx 7900 \frac{\mathrm{kg}}{\mathrm{m}^{3}}$ ) sediment at the bottom of the tube, whereas low-density oil $\left(\rho \approx 800 \frac{\mathrm{kg}}{\mathrm{m}^{3}}\right)$ forms an upper layer in the tube. By discarding the upper layer, the oil can be eliminated. This procedure of adding DI water and centrifuging is repeated 3 times to make sure that all the oil was eliminated. Centrifugation time $t_{\mathrm{i}}$ during each iteration was decreased, as discussed below. The suspension of particles in DI water $(3 \mathrm{ml})$ is then collected for further analysis. The diagram of the process is shown in Fig. 1.

For the centrifugation, a Thermo Scientific SL 16R centrifuge (Thermo Fisher Scientific, Waltham, MA, USA) was used at $10,000 \mathrm{rpm}$ and $40{ }^{\circ} \mathrm{C}$, resulting in the relative centrifugal force $R C F=\frac{r \omega^{2}}{g}=10,947$ in the particle isolation procedure.

The required centrifugation time $t_{\mathrm{i}}$ at each iteration was estimated by assuming that the particles are spherical and that there are only centrifugal, buoyancy and viscous drag forces (Stokes drag) acting on the particles. A force balance leads to the following expression for the time needed for a single particle to sediment at the bottom of the tube [32]:

$t=\frac{9}{2} \frac{L \mu}{R C F \times g\left(\rho_{\mathrm{p}}-\rho_{1}\right) R^{2}}$,

where $g$ is the gravitational acceleration, $\rho_{\mathrm{p}}$ and $\rho_{1}$ are the density of the particle and liquid, $R$ is the radius of the particle, $\mu$ is the viscosity of the liquid and $L$ is the particles traveling distance. In order to get a simple equation, it was assumed that the interaction between the particles is negligible. It should be noticed that the interaction of particles may lead to agglomeration [33, 34] and increased mass of the formed agglomerate. This would lead to higher centrifugal force and decrease the centrifuging time. Therefore, the discussed assumption results in upper bound for the centrifugation time. It should also be noted that the centrifugation time obtained by Eq. (2) was used only as an indication, and in the separation procedure even longer time was used, as discussed below.

If it is assumed that the tube is fully filled by oil $(\mu \approx 55 \mathrm{cP})$, then at least $12 \mathrm{~h}$ are required for the spherical 
Fig. 1 Schematic diagram of the particle isolation procedure

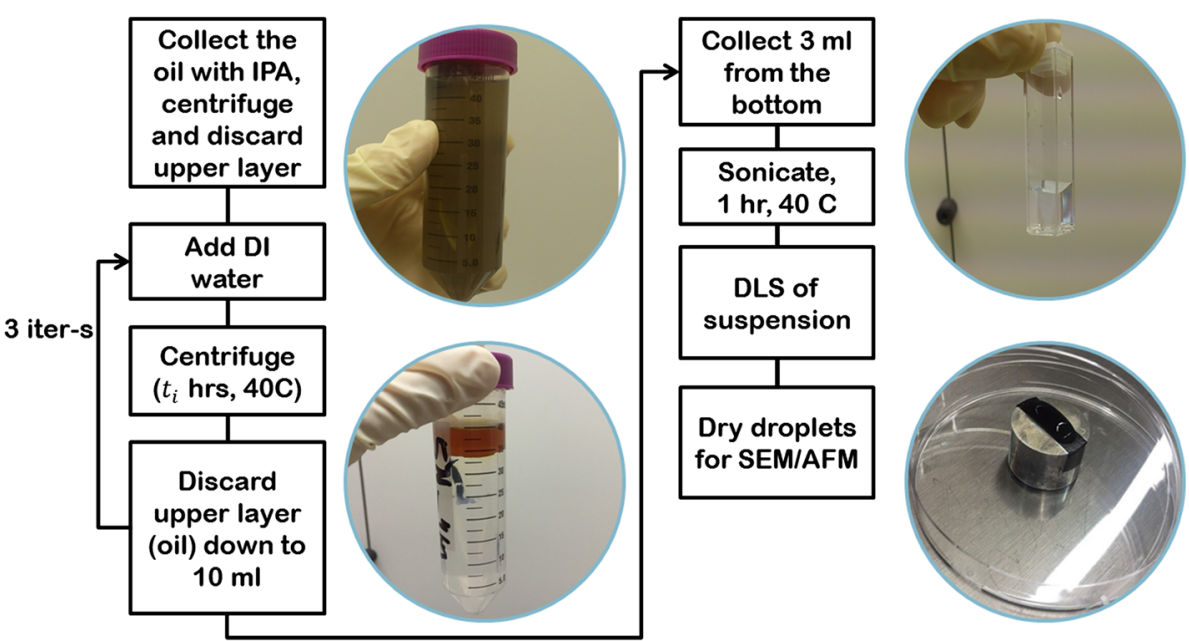

particles with radius larger than $25 \mathrm{~nm}$ to sediment at the bottom of the tube according to (2). Since the calculated time is only an approximation, the first centrifugation was done for $t_{1}=15 \mathrm{~h}$. After the first centrifugation, most of the oil is eliminated and it can be assumed that the viscosity of the left liquid is equal to the viscosity of DI water of $1 \mathrm{cP}$. In this case, for the same size of particles to sediment, theoretically only $15 \mathrm{~min}$ are required. Therefore, after the first iteration, consequent centrifugation times can be significantly decreased. The centrifugation times were taken to be $t_{2}=4$ and $t_{3}=2 \mathrm{~h}$.

\subsection{DLS Measurement}

For the DLS measurements, a Zetasizer Nano ZS system (He-Ne laser of $633 \mathrm{~nm}$ wavelength, Malvern, UK) with $1.5 \mathrm{ml}$ of particle suspension was used. Measurements were taken at $25{ }^{\circ} \mathrm{C}$. The result of the measurement is an equivalent hydrodynamic diameter [35]. Equilibration of the sample before the measurements was performed for $120 \mathrm{~s}$. The diameter of the particles was measured with refractive index of 2.87 and 0.58 for steel and brass, which corresponds to pure iron and copper at $633 \mathrm{~nm}$ wavelength [36]. Repeated DLS measurement on the same sample at various times after particles isolation did not reveal significant variation of the size, indicating stability of the suspension. It was also found that the increase in the sonication time after centrifugation did not affect the particles size measurement.

\subsection{Preparation of Samples for SEM/EDS and AFM Measurements}

After DLS analysis, samples were further analyzed using SEM/EDS and AFM.

SEM measurements coupled with EDS were taken to validate the particle isolation procedure by analyzing elemental composition of debris. Suspensions of particles were dried on a carbon tape for 1 day in a vacuum oven at $40{ }^{\circ} \mathrm{C}$. Next, a JEOL JSM-6400 Scanning Microscope (JEOL, Tokyo, Japan) coupled with EDS was employed.

AFM measurements were taken to obtain 3D information about the particles and to build the distribution of particles size. Samples were dried on a glass plate for 1 day in a vacuum oven at $40{ }^{\circ} \mathrm{C}$. A glass plate was used since the wear particles can be visually identified on the glass plate using a microscope. Measurements were taken using a FlexAFM microscope (Nanosurf AG, Liestal Switzerland) in a dynamic mode. The scan window was taken to be $20 \mu \mathrm{m} \times 20 \mu \mathrm{m}$ with $1024 \times 1024$ measurement points. Silicon tips (ACLA) with the cantilever stiffness of $42 \mathrm{~N} / \mathrm{m}$ and tip radius $>6 \mathrm{~nm}$ were used.

The AFM height profiles were analyzed in MATLAB to identify wear particles. A roughness profile of the clean glass substrate is shown in Fig. 2. The roughness of the glass plate was found to be approximately $5 \mathrm{~nm}$, which means that $99 \%$ of its surface heights are within $30 \mathrm{~nm}$. Therefore, within the range of $30 \mathrm{~nm}$, it is not possible to distinguish the particles from the substrate on AFM profile.

An example of an AFM profile of the substrate with particles on it is shown in Fig. 3. On the left image, the particles can be visually identified. On the cross section, clearly, the peaks correspond to the particles, since the glass plate is much smoother.

A height threshold was therefore introduced to ensure that the roughness of the substrate and possible noise of the measurement are excluded in the analysis. An example of the initial AFM height profile and identified particles using $50 \mathrm{~nm}$ height threshold is shown in Fig. 4. Based on this information, the distributions of width, length and thickness of the particles were obtained. The width and length were defined as the longest dimension of the square which encompasses the particle, being the width the smallest dimension and the thickness was defined as the deepest point of a particle. 
Fig. 2 AFM height profile and its cross section for clean glass substrate $\left(R_{q} \approx 5 \mathrm{~nm}\right)$

Fig. 3 AFM height profile of the plate with dried particles suspension $R_{q} \approx 50 \mathrm{~nm}$
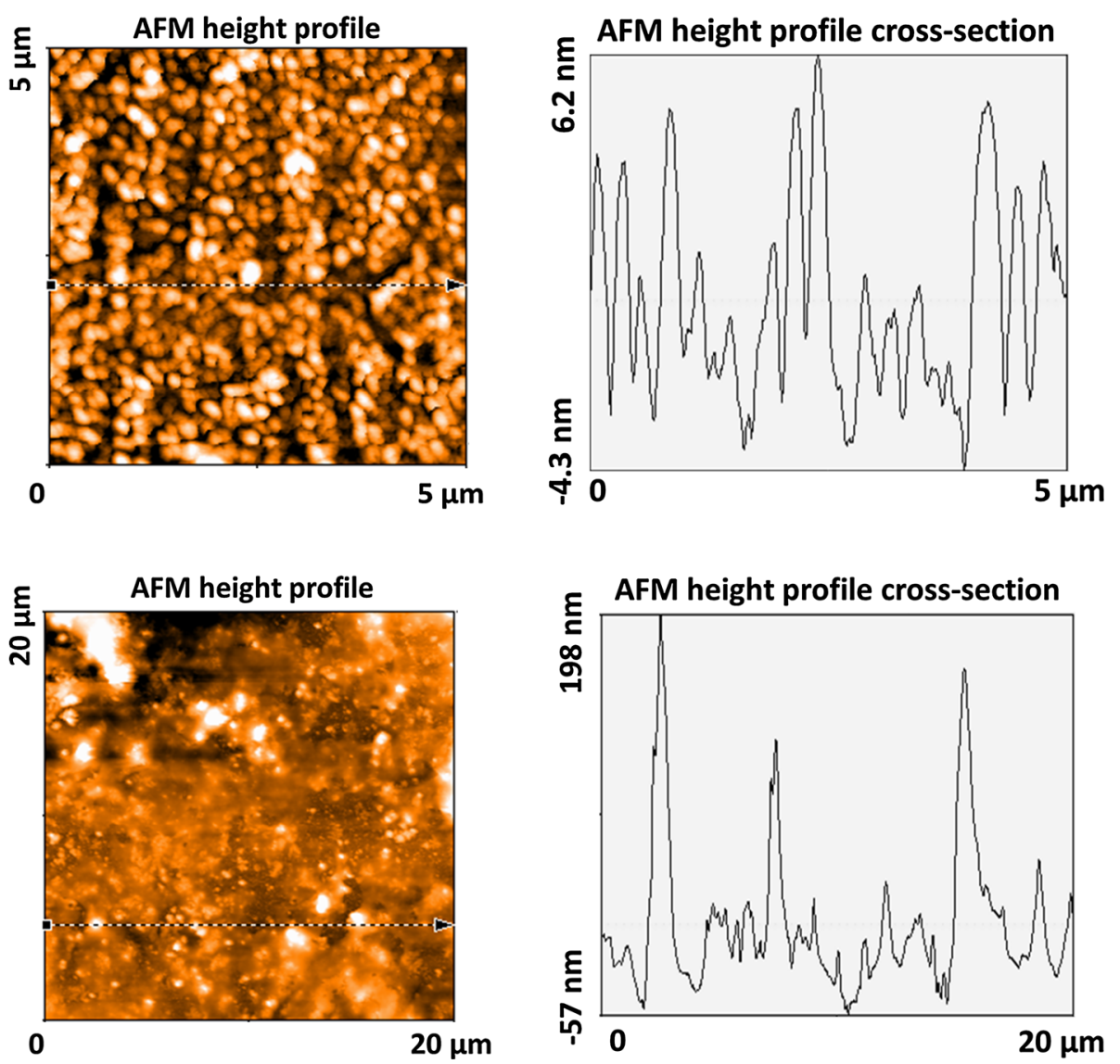

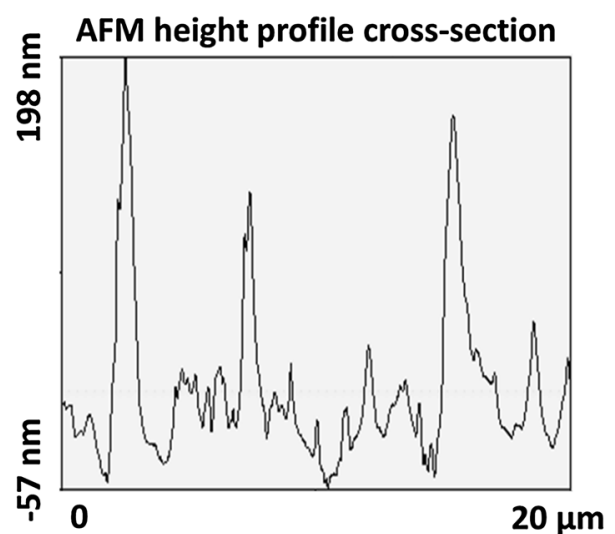

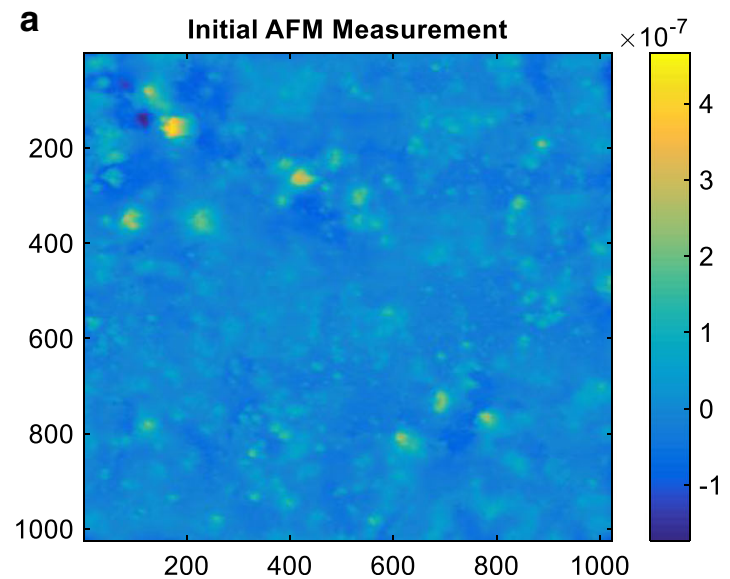

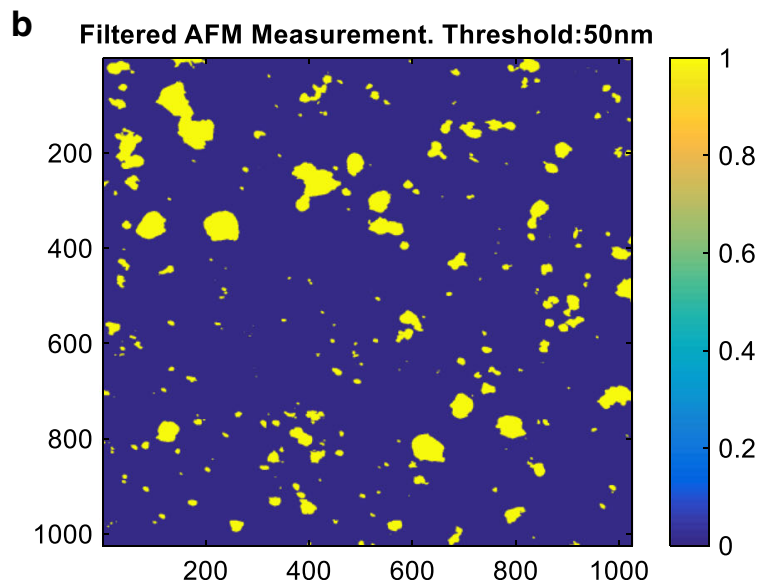

Fig. 4 Identification of particles with height threshold (50 $\mathrm{nm}$ threshold)

\section{Results and Discussion}

\subsection{Friction and Wear Volume Measurement}

During the wear tests, the coefficient of friction was recorded and wear volumes were estimated using surface profile measurements as described above. It was found that the wear volume of the cylinder was three orders of magnitude less than the wear of the steel disk at $10 \mathrm{~N}$ load. In case of the brass disk, it was found that the brass transfer film formed on the surface of the cylinder and no significant wear of the cylinder was detected. Therefore, it was assumed that most of the analyzed particles originated from the disks.

The regime of lubrication was assessed using $\lambda=$ $h_{\min } / R_{q}$ [37], where $h_{\min }$ is the minimum film thickness obtained by Dowson-Higginson equation [38, 39]. For the 
case of steel-steel contact at $2.5 \mathrm{~N} \operatorname{load} \lambda=0.051$, and since $\lambda<0.1$ the boundary lubrication regime was encountered [40]. Under higher loads or with brass material, $\lambda$ is even smaller, and therefore, all the considered tests operated in boundary lubrication regime. It can be seen from Fig. 5 that the coefficient of friction does not change significantly with load for both material combinations. This suggests that the dominating wear mechanism will also be the same for all measurements. The friction with brass disk is approximately twice as high as with the steel disk.

There was no significant variation of the friction with temperature. This was to be expected, since, in the considered range of temperatures, the mechanical properties of the materials are not influenced significantly. A COFtemperature dependence is also not expected from a possible formation of a tribo-layer. In these tests, no extreme pressure and anti-wear additives are used. However, even for additive-rich oils, in the range of temperatures considered, friction will not depend on temperature [41]. In this range of temperatures, the oxide type remains the same [42].

Results of wear volume measurements are shown in Fig. 6. For both materials, there is a linear relation of wear volume with load. The wear of the brass is higher, since it has twice as high COF as the steel. The wear volume of brass as a function of load increases stronger than that of steel disk.

Wear coefficients were obtained from the Archard's wear equation in the following form [43]:

$W=k \times F \times s$,

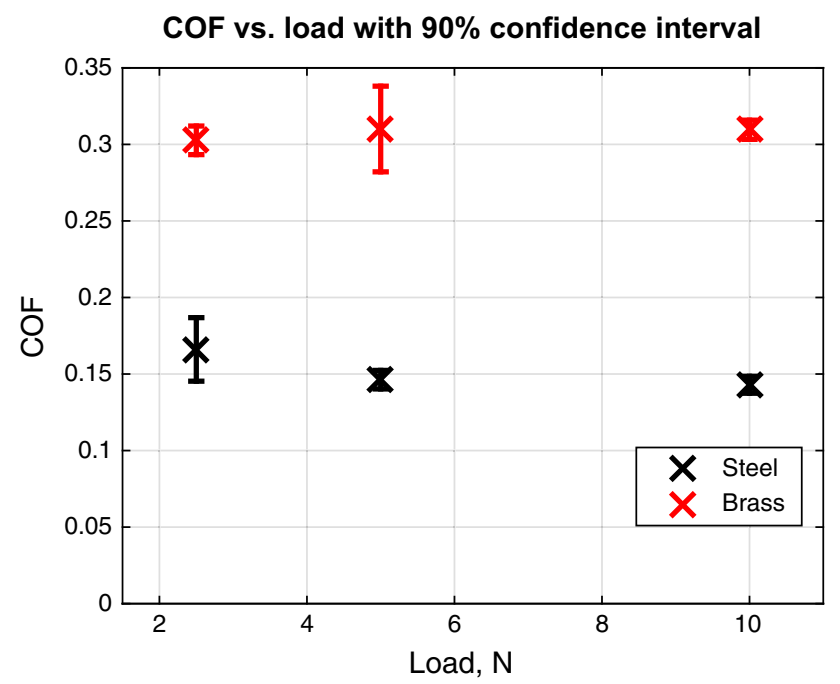

Fig. 5 Coefficient of friction as a function of load for steel and brass disks

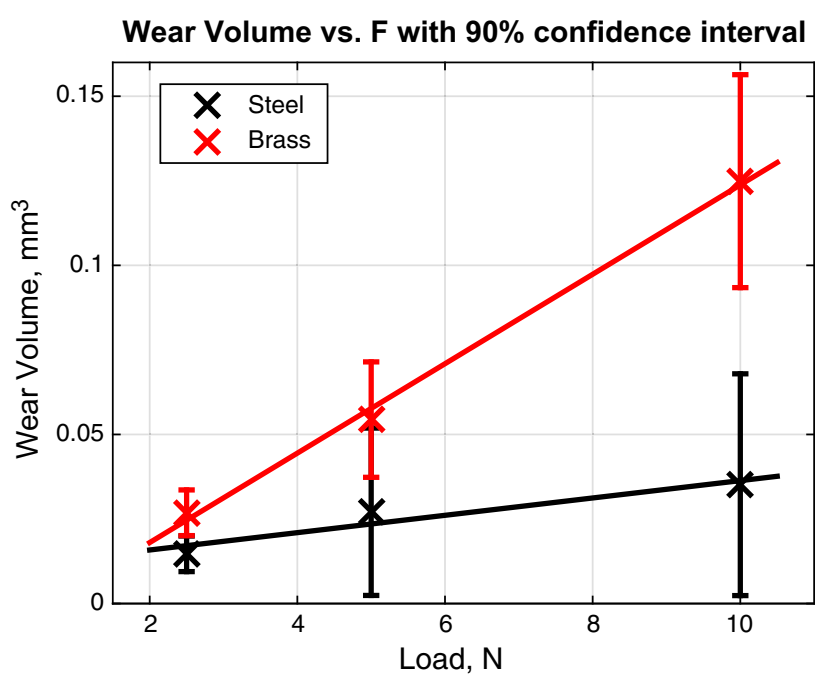

Fig. 6 Wear volume variation with load

where $F$ is the applied normal load, $s$ is the sliding distance, $W$ is the wear volume and $k$ is specific wear rate. It was found that the mean value of $k$ for steel disks was around $350 \times 10^{-7} \mathrm{~mm}^{3} / \mathrm{Nm}$ and for brass disks $800 \times$ $10^{-7} \mathrm{~mm}^{3} / \mathrm{Nm}$.

From Fig. 7, it can be clearly seen that for both materials there is a slight change of the specific wear rate coefficient with load. For brass, there is a slight increase, whereas for steel a decrease.

The evolution of surface roughness during the wear test was studied as a function of load in a following form:

$\delta R_{q}=\frac{\left(R_{q \text { worn }}-R_{q \text { initial }}\right)}{R_{q \text { initial }}} \times 100 \%$,

where $\delta R_{q}$ is the increase in roughness in $\%, R_{q \text { initial }}$ and $R_{q \text { worn }}$ are roughness of initial and worn surfaces.

Variation of $\delta R_{q}$ with load is given in Fig. 8. It can be seen that for $2.5 \mathrm{~N}$ load, the roughness after the test somewhat decreased for both materials. However, for the loads of 5 and $10 \mathrm{~N}$, the "worn roughness" is significantly higher than the initial for brass. In case of steel, the worn roughness is lower than the initial in all cases, so the surface was smoothened during the wear test.

\subsection{SEM/EDS Measurements}

The major goal of the particle isolation procedure is to collect wear particles from the oil for further analysis. Firstly, it was confirmed by the presence of iron in the collected particles that they originate from the base material (in this case of the steel-steel) sliding pair.

The SEM/EDS results are shown in Fig. 9. A SEM image of a particle is shown in Fig. 9a and the 


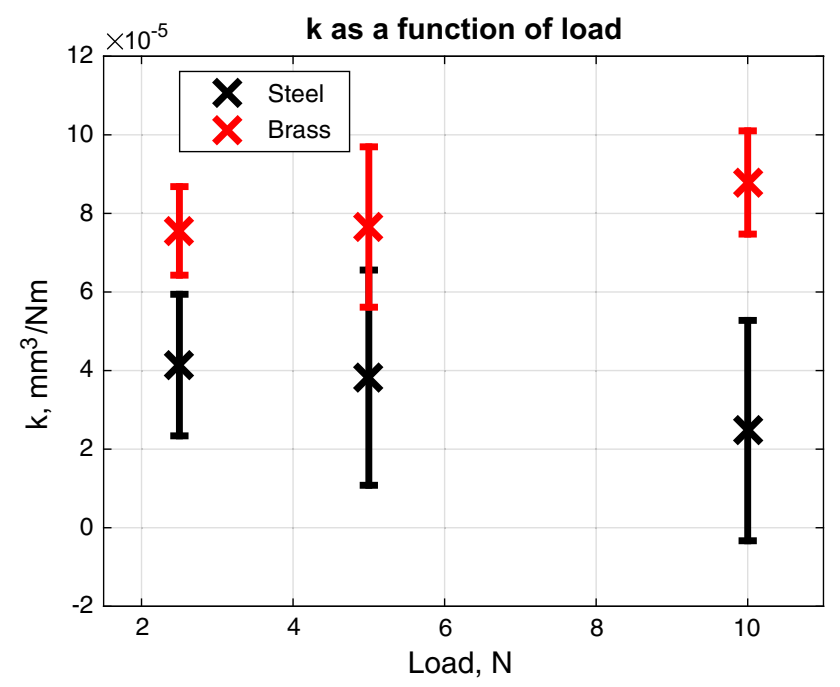

Fig. 7 Specific wear rate coefficient

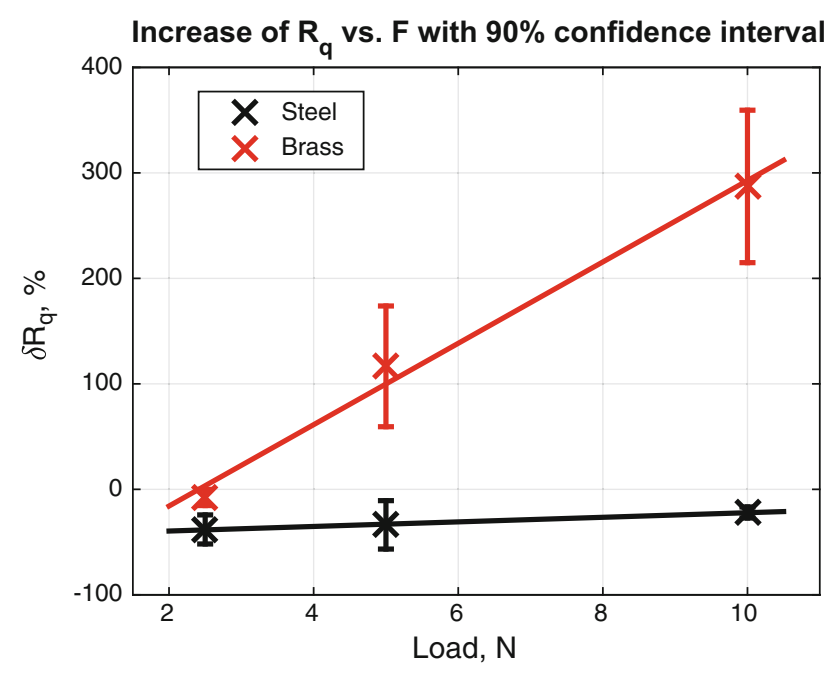

Fig. 8 Increase in roughness with load

corresponding EDS element mapping in Fig. 9b. It can be clearly seen that iron is present in the particle. There is also $\mathrm{C}$ (carbon) present, which originates from the carbon tape used for preparation of the sample. The presence of oxygen shows that the particle is oxidized. The measurement of elemental composition was performed for several particles, and iron was found in all of them, and therefore, it can be concluded that the wear particles can be collected using the developed isolation procedure.

\subsection{DLS Measurements of the Particles}

Significant effort in this study was devoted to the DLS analysis of the size of the wear particles. First, a measurement (including the particle isolation procedure) was taken with fresh oil which confirmed that no particles were observed. Coupled with the SEM/EDS measurements, it proves that the isolation procedure does not introduce artifacts in the measurement.

Figure 10 shows the relation between the characteristic particles radius (the radius obtained from DLS) on load. This figure shows that there is a trend in the particles radius with load in the case of steel. The difference of the mean radius at $10 \mathrm{~N}$ and at $2.5 \mathrm{~N}$ is approximately $7 \%$. This does not apply to the case of the brass disks. Apparently, the size does not depend on the load here. The particles have smaller radius roughly by $20-40 \mathrm{~nm}$ than formed in a steel-steel pair.

Figure 11 shows the characteristic radius as a function of temperature for the case of steel disks. Rubbing at different temperatures gives wear particles with the similar radii.

By dividing the total wear volume by a volume of a characteristic wear particle, the total number of particles can be found. The results are shown in Fig. 12a.

Dividing Archard's Eq. (3) by a volume of a characteristic particle results in the following relation:

$N_{\mathrm{p}}=k_{N} \times F \times s$,

where $N_{\mathrm{p}}$ is the number of particles and $k_{N}=k / W_{\mathrm{p}}$ is the specific wear particle coefficient with $W_{\mathrm{p}}$ being the volume of characteristic particle.

The calculated values of $k_{N}$ as a function of load are shown in Fig. 12b. It should be noted that $k_{N}$ does not depend on load significantly for brass. This makes it convenient to estimate the total number of particles without knowing the mean size of a particle in similar conditions.

\subsection{AFM Measurements of Steel Wear Particles}

After the DLS measurements, several samples from the tests with steel disks under 2.5 and $10 \mathrm{~N}$ load were analyzed using AFM to obtain 3D information regarding particle size. Overall, 2500 particles were analyzed with nearly 500 particles for the samples at $10 \mathrm{~N}$ and 2000 particles for the samples at $2.5 \mathrm{~N}$. Based on these measurements, the distributions of length, width and thickness were built.

In order to compare the results of AFM measurements with those obtained by DLS, equivalent radii of the particles were calculated. These are based on the radius of a spherical particle with the same volume given by:

$R_{i}=\left(\frac{V_{i}}{\pi} \frac{3}{4}\right)^{1 / 3}$,

where $R_{i}$ is the equivalent radius of the particle and $V_{i}$ is its volume obtained from AFM analysis. The probability 

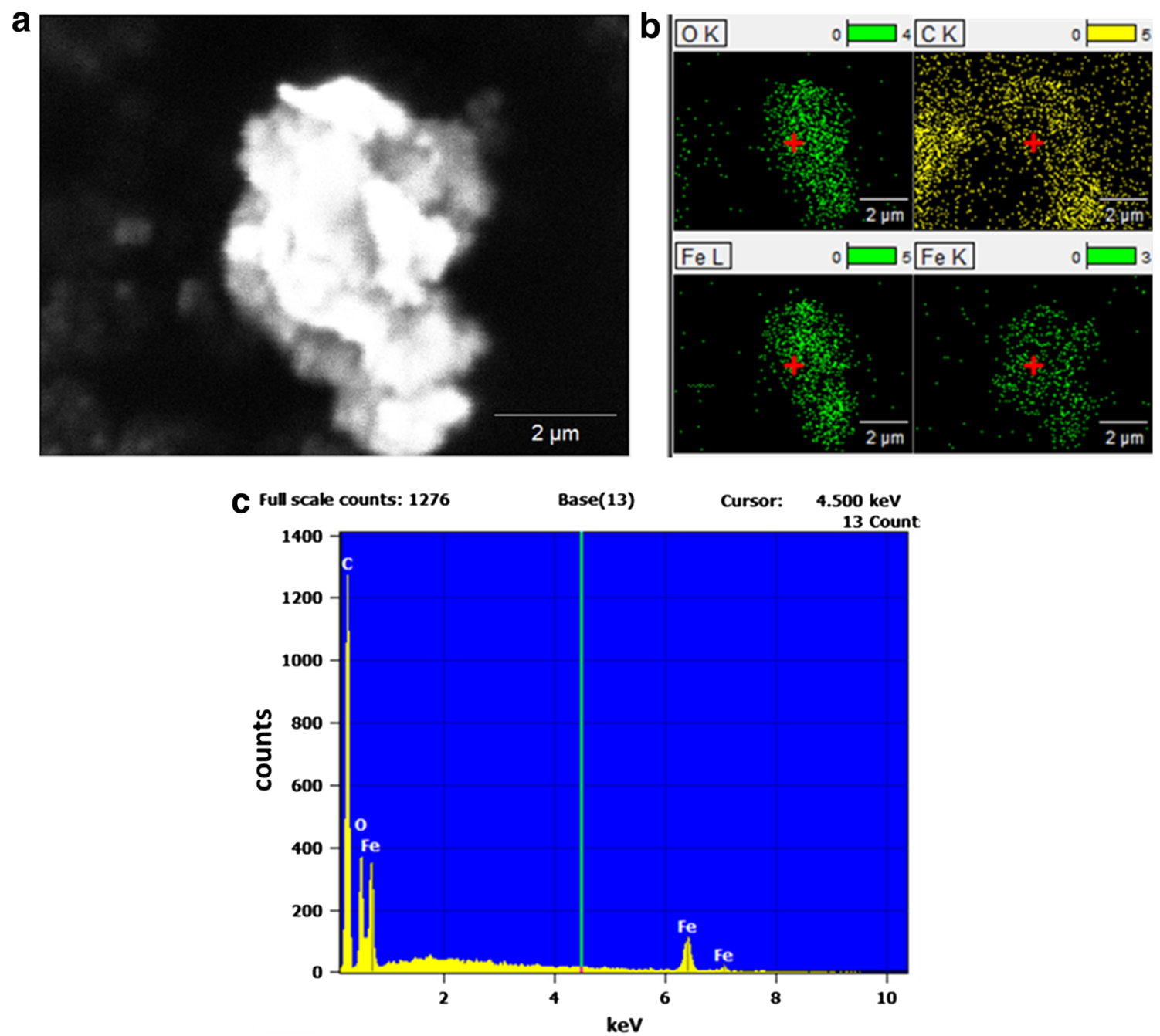

Fig. 9 SEM/EDS results obtained for steel disk: SEM image (a), element mapping (b) and EDS spectrum (c)

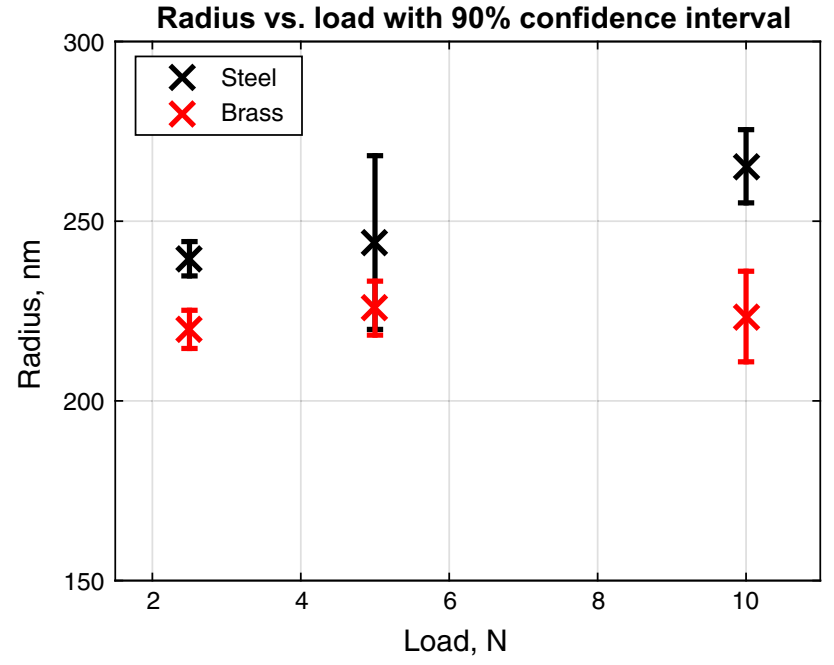

Fig. 10 Radius of a wear particle as a function of load

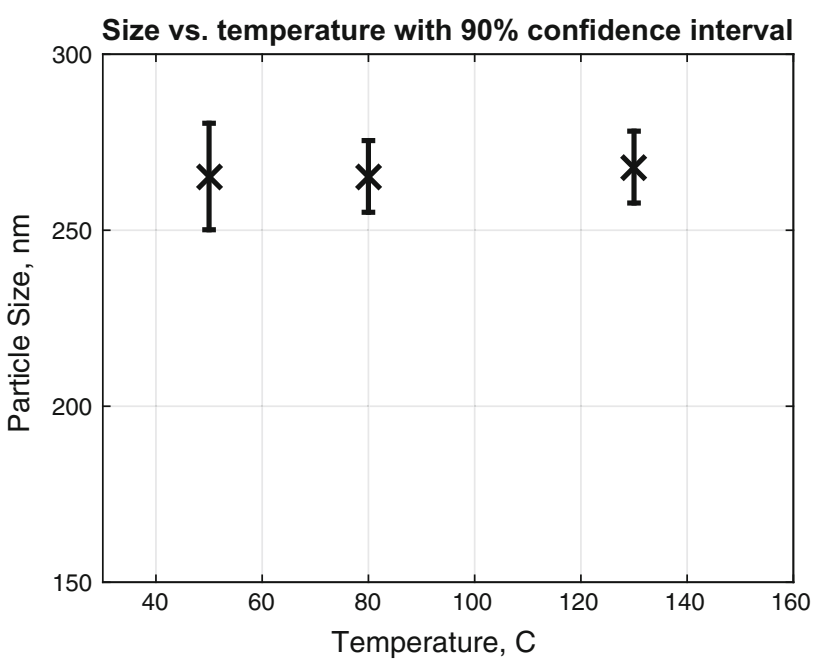

Fig. 11 Radius of a wear particle as a function of temperature, steel disk 

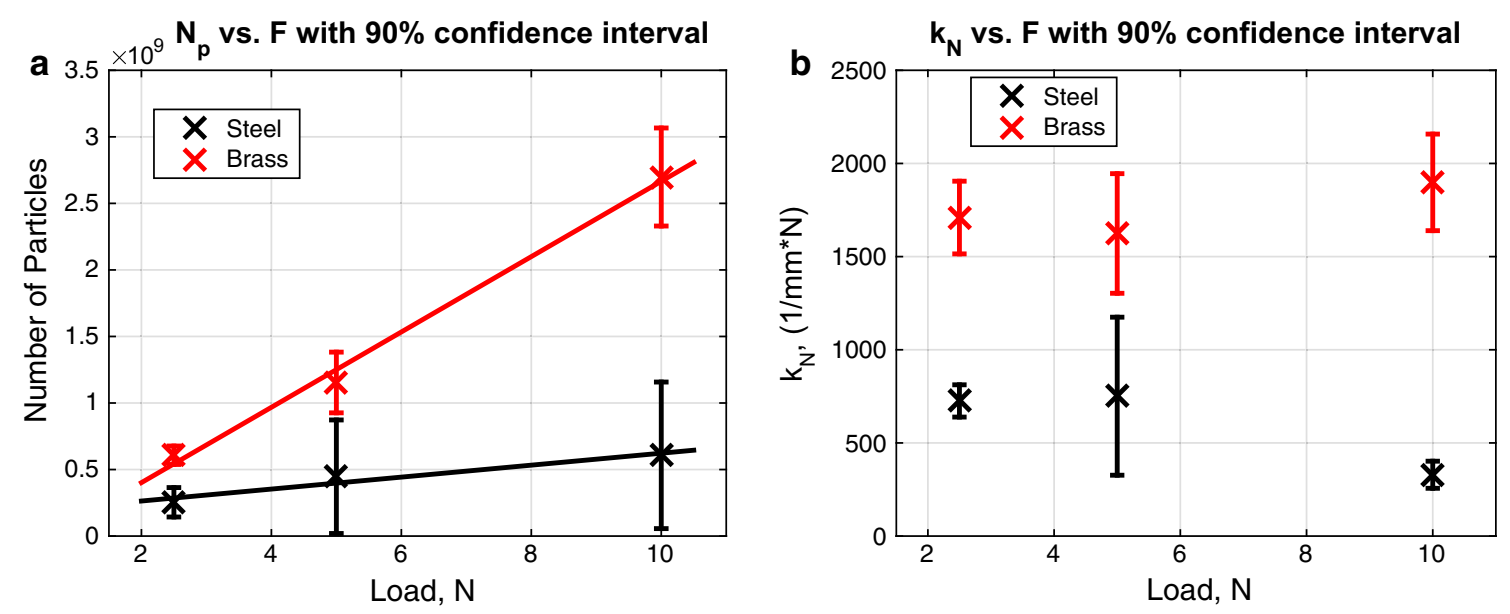

Fig. $12 N_{\mathrm{p}}(\mathbf{a})$ and $k_{N}(\mathbf{b})$ as a function of load

distributions of the equivalent radius of particles obtained under $10 \mathrm{~N}$ load are given in Fig. 13.

The number-weighted mean value of the equivalent radius was found to be around $175 \mathrm{~nm}$, whereas the volume-weighted mean value was found to be $358 \mathrm{~nm}$. It means that most of the volume is composed by a small amount of relatively large particles, whereas the number of smaller particles is dominant. DLS results for the same sample showed an equivalent radius of approximately $265 \mathrm{~nm}$. The tendency of the DLS approach toward higher values of particle's size compared to AFM was already reported in the literature [44]. The equivalent hydrodynamic diameter may be biased toward higher radius particles, since it is proportional to the scattered intensity, and for small particles, it is proportional to the sixth power of the radius $[35,44]$. It can be noticed that the distribution of the particles size is not Gaussian. However, the distribution is mono-modal. This indicates that there are only limited wear particles originating from the cylinder, otherwise it could be expected to get at least two peaks in the distribution, corresponding to the disk and to the cylinder.

The situation is similar for the particles generated at $2.5 \mathrm{~N}$, see Fig. 14. The total wear volume is dominated by the small amount of large particles, and this effect is even more pronounced compared to the samples tested at $10 \mathrm{~N}$.

The distributions of the length, width and thickness of the particles were also obtained for both loads and both materials. Qualitatively similar distributions were obtained, and for brevity only results at $10 \mathrm{~N}$ for steel disks are shown, see Fig. 15. The thickness of the particles was found to be approximately 4-5 times less than the width, which means that the particles are flat. It can be noticed
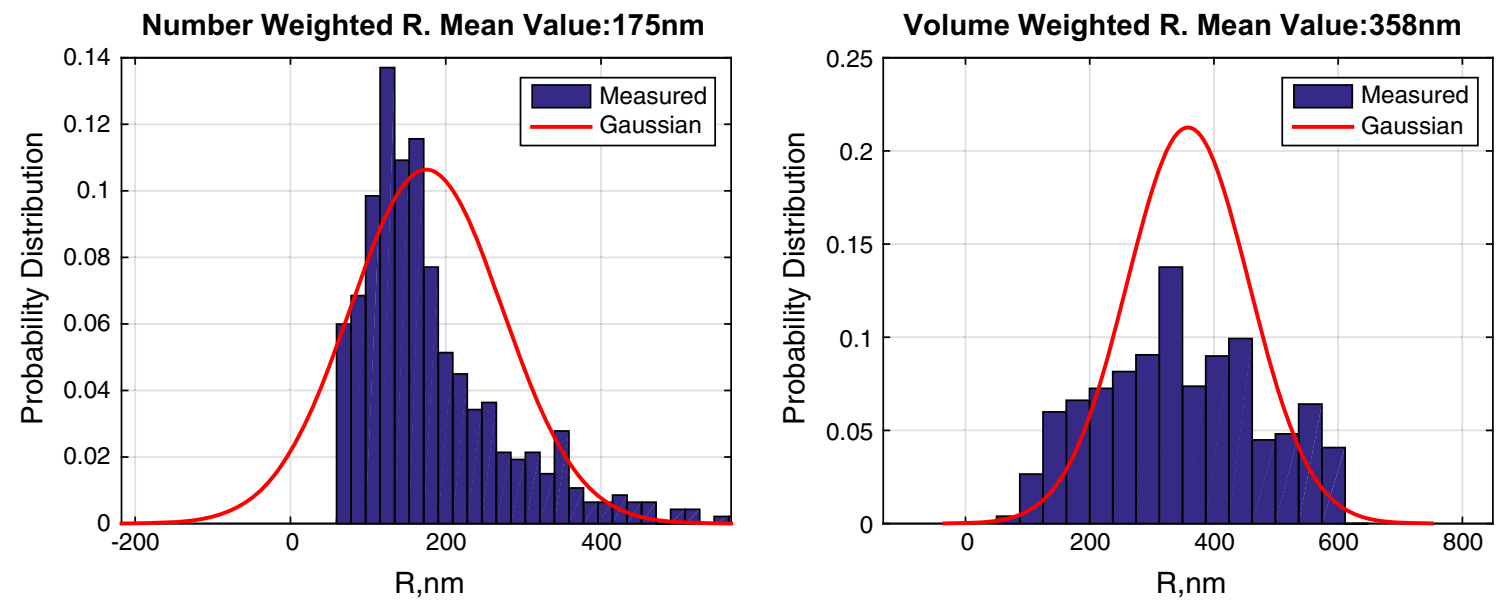

Fig. 13 Number- and volume-weighted equivalent radius probability distributions. Steel disk, 10 N, 467 particles total 

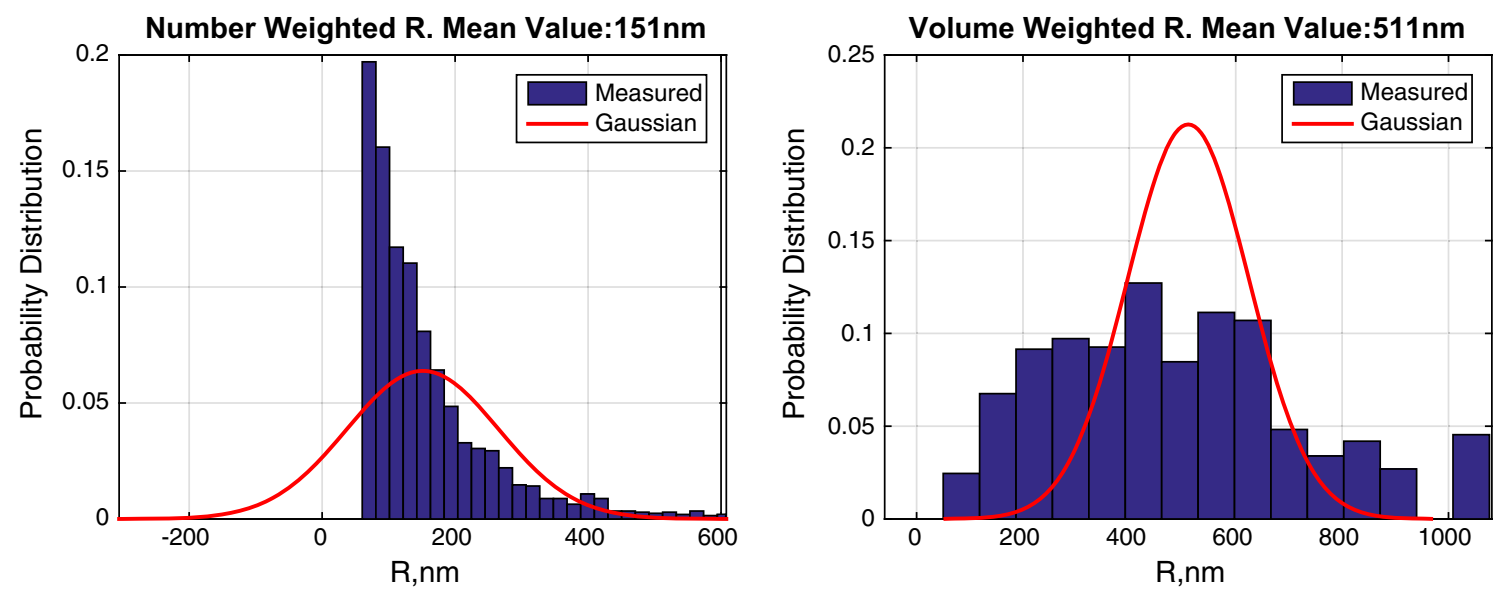

Fig. 14 Number- and volume-weighted equivalent radius probability distributions. Steel disk, 2.5 N, 1500 particles total

that the distributions are negatively skewed and diverge from the Gaussian distribution, i.e., there are relatively many particles with a small radius.

It is also necessary to compare the surface area-weighted mean radius of the particles. Comparison of the distributions is shown in Fig. 16. In both cases, the number-weighted mean radius is smaller than the surface area weighted. This fact and the fact that the shape of the distributions show that most of the surface area comes from the large particles, although the number of the small

Number Weighted Length. Mean Value:747nm
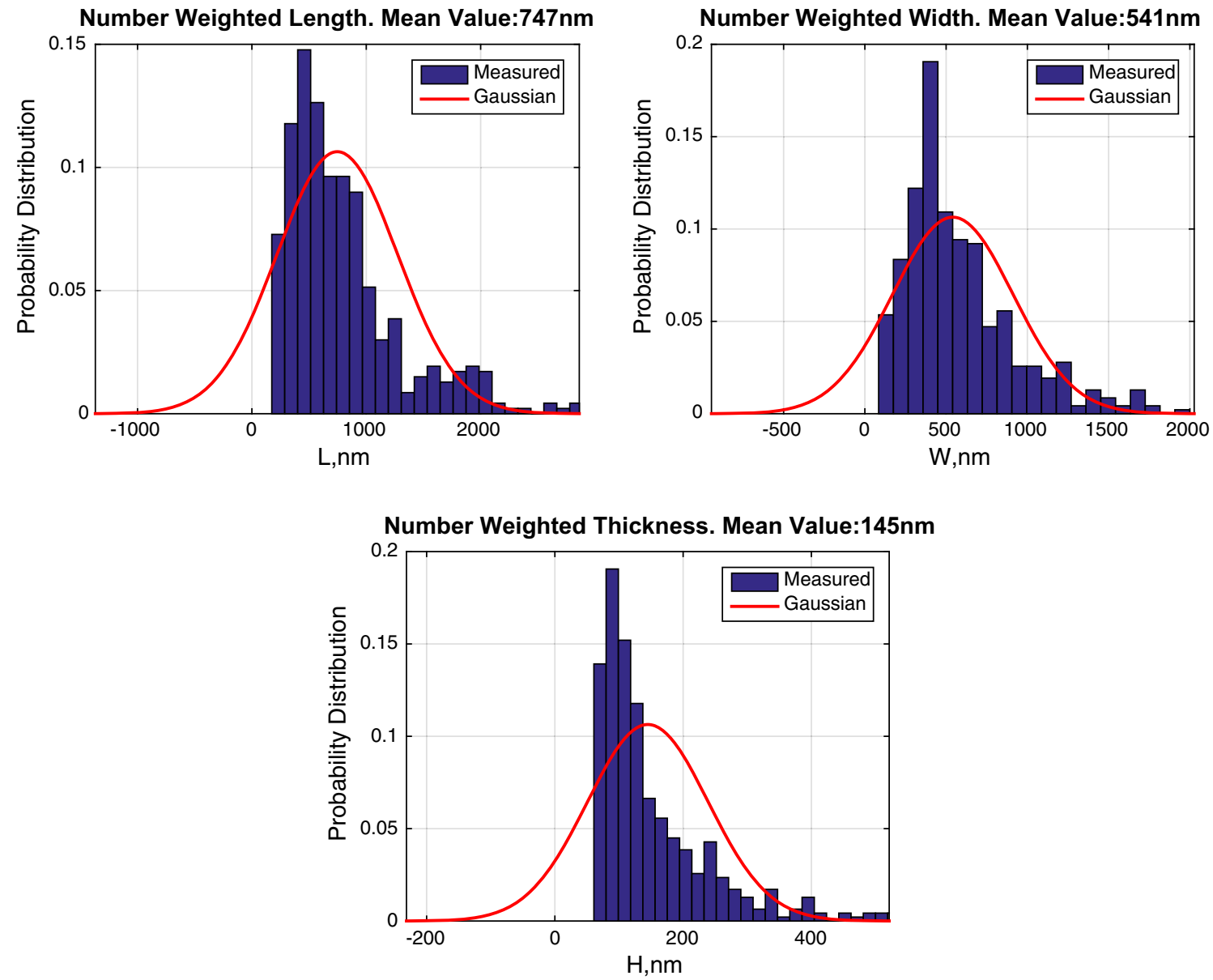

Fig. 15 Distribution of particles length, width and thickness, steel disk, $10 \mathrm{~N}$ 
particles according to number-weighted distributions gets larger. Therefore, even though the number of small particles gets higher, their impact on the total surface area or wear volume becomes less.

Finally, comparison of the DLS and AFM data is shown in Fig. 17. A slight trend of the increase in the particles size with load can be found from both DLS and AFM results for steel disk. In case of brass disks, DLS results do not show such trend and the obtained radius stays approximately constant with load. The AFM results show smaller particles sizes due to bias of DLS toward larger particles. At the same time, AFM also has a bias, although it is apparently less expressed. Due to the height threshold, a number of small particles are not considered, next to the fact that in AFM only the upper contour is obtained, and thus, volume of undercuts is added to the wear particles. Since the number of these particles is relatively large, a large number of particles are not considered, which leads to the bias of AFM results toward larger sizes. This bias is more pronounced for low loads, when the size of the particles is close to the height threshold.

\subsection{Discussion}

The main challenge of the wear particles analysis lies in the preparation of the samples and related artificial filtering. There is a possibility of the wear particles to aggregate into larger clusters, and sometimes these clusters may not be broken by the sonication [29]. Therefore, the obtained results can be considered as the upper bound estimation for the wear particles size. The particle isolation procedure also introduces a filtering of particles smaller than approximately $25 \mathrm{~nm}$ radius due to the limited centrifugation time. It should also be noted that hypothetically there is no minimum wear particles size (the theoretical smallest possible wear particle is an ion).
On the other hand, there is no need in putting efforts to capture smaller particles during the centrifugation, since both DLS and AFM techniques introduce coarser filtering. In case of DLS, the particles with the radius smaller than $100 \mathrm{~nm}$ will have significantly less weight in the determination of the mean value of the wear particle. This results in relatively large mean particle radius observed by DLS. In case of AFM, the filtering is introduced explicitly through the height profile threshold. This threshold is necessary to distinguish the particle from the background roughness and possible noise. In this work, the threshold was taken to be $50 \mathrm{~nm}$. Due to filtering, it is also clear that the obtained values of the wear particles size are larger than the actual sizes in case of both DLS and AFM analyses.

On the other hand, the contribution of the smallest particles to the overall wear volume or surface area of worn particles sometimes can be limited. It can be argued that in the presence of large particles which make most of the wear volume, the mechanical damage from wear particles can be ascribed to these large particles. They are capable of making relatively large dents, scratches and other mechanical damage, as in case of large abrasive particles [45]. Smaller particles, within the range of the surface roughness, are going to create relatively minor damage $[46,47]$, since they may carry less load. The fact that the number of small particles is high but their contribution to the total wear volume is low is found by the comparison of the number- and volume-weighted distributions obtained using AFM, see Figs. 13 and 14. Moreover, with the decrease in the overall mean particles size, this effect becomes even more pronounced. Wear particles also play significant role in chemical processes, such as chemical deterioration of the grease in bearings [11]. The degree of catalytic effect of wear particles on chemical reactions is mostly determined by the total surface area of the particles
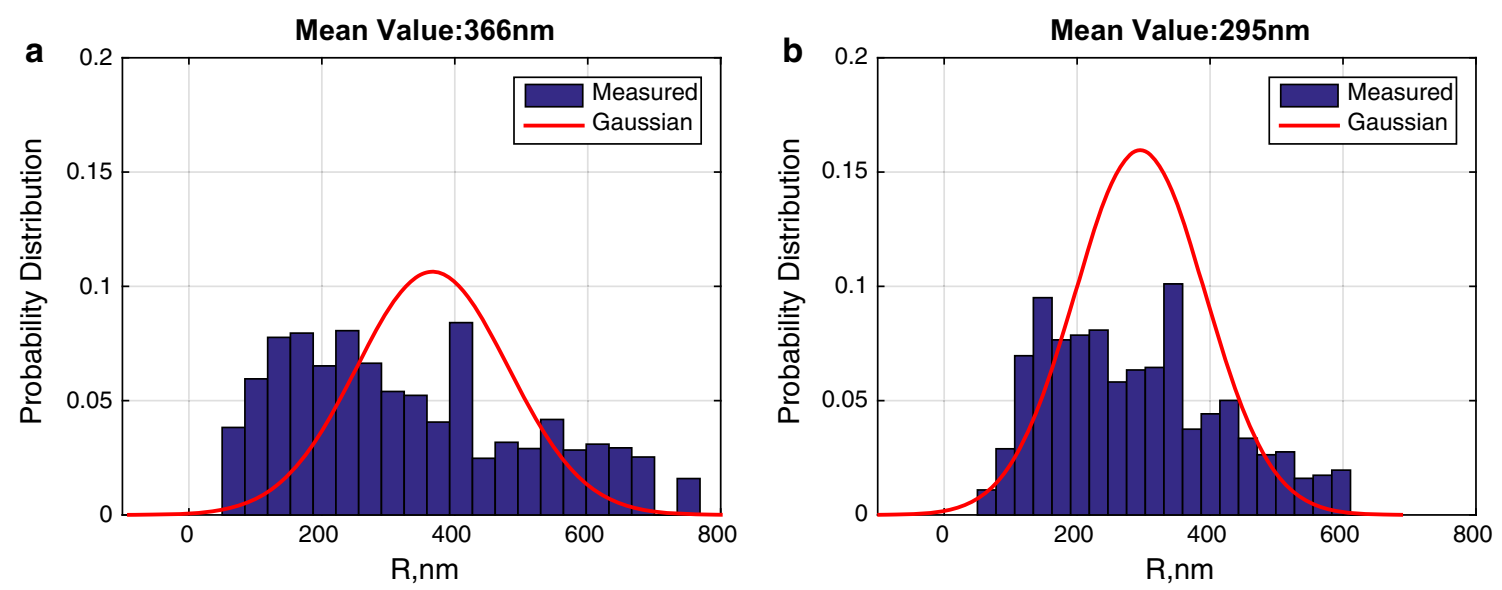

Fig. 16 Surface area-weighted wear particle radius at $2.5 \mathrm{~N}$ (a) and $10 \mathrm{~N}$ (b) 

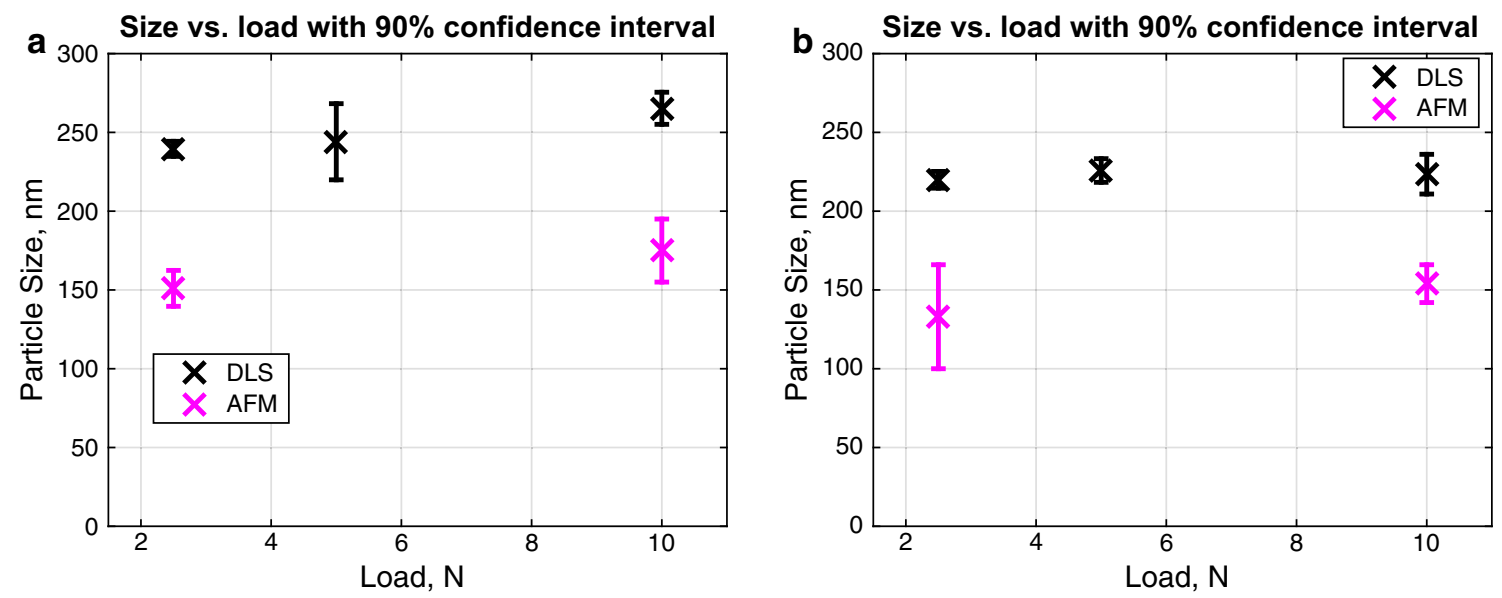

Fig. 17 Comparison of DLS and AFM results for steel (a) and for brass (b)

rather than by their volume [48]. From the AFM results regarding the surface area-weighted particle size, it can be concluded that the contribution of the small particles to the total surface area of the worn particles decreases rapidly with a decrease in the particles size, see Fig. 16. In addition, it must be noted that relatively large particles will always be generated in the system in the running-in stage of the wear process [8].

Based on the results of AFM measurements, a slight dependence of the particles size on load can be observed for both materials. This behavior is confirmed by DLS for steel samples. It should be noted that with the decrease in the applied load, the size of the particles becomes smaller and the effect of the filtering (both by AFM and DLS) becomes more pronounced. Therefore, the actual dependence of the size on load may be more noticeable, especially at lower loads. Based on DLS results for brass disks, it is not possible to draw a conclusion on the relation between load and size. This may be due to resolution of the DLS and only slight changes of the particles size.

AFM measurements also revealed that the shape of particles is characterized by a high aspect ratio (around 4-5) meaning that the particles are flat, as it is frequently reported in the literature [1, 49]. The formation of flat particles suggests that severe plastic deformation takes place on the surface and subsurface in the presence of a tangential load. The wear particle may form in such regions $[50,51]$.

Specific wear rates obtained in these measurements indicate a running-in stage of the sliding friction [52]. It is suggested that most of the wear volume is generated during this initial stage [22, 53], and therefore, it can be concluded that most of the particles are also formed during the running-in stage. In the present work, the values of $k_{N} \approx$ $330-750 \frac{\text { particles }}{\mathrm{mmN}}$ for steel and $k_{N} \approx 1600-1900 \frac{\text { particles }}{\mathrm{mmN}}$ for brass were obtained. These values then can be considered as characteristic to the running-in stage of the discussed system. It should be noted that calculation of the number of particles based on DLS is approximate and underestimates the real number of particles due to bias toward large particles.

\section{Conclusions}

The method for collecting and analyzing wear particles from lubricated wear experiments was developed, validated and discussed. The procedure can be applied for the preparation of samples for DLS, SEM/EDS and AFM measurements.

Based on DLS measurements, it was shown that the radius of particles is in the range of $230-360 \mathrm{~nm}$ for both materials. AFM results showed the size to be in the range from 133 to $175 \mathrm{~nm}$. A slight dependence particles size on load was obtained both by AFM for both materials and by DLS for steel samples. AFM measurements showed monomodal non-Gaussian size distribution of flat particles with aspect ratio in the order of $4-5$. The number of particles formed per sliding distance and unit of load were estimated to be in the range from 150 and $750 \frac{\text { particles }}{\mathrm{mm} \mathrm{N}}$ for steel and approximately $1600-1900 \frac{\text { particles }}{\mathrm{mmN}}$ for brass, In case of steel-steel pair, wear debris was mostly composed of iron.

Obtained data can be used for validation of the theoretical wear particles formation models.

Acknowledgments The authors would like to thank SKF Engineering and Research Centre, Nieuwegein, the Netherlands, and Bosch Transmission Technology BV, Tilburg, the Netherlands, for providing technical and financial support. We would like to thank Mr. A. J. C de Vries, SKF Director Group Product Development for his permission to publish this work. This research was carried out under Project 
Number M21.1.11450 in the framework of the Research Program of the Materials Innovation Institute M2i.

Open Access This article is distributed under the terms of the Creative Commons Attribution 4.0 International License (http://crea tivecommons.org/licenses/by/4.0/), which permits unrestricted use, distribution, and reproduction in any medium, provided you give appropriate credit to the original author(s) and the source, provide a link to the Creative Commons license, and indicate if changes were made.

\section{References}

1. Bhushan, B.: Principles and Application of Tribology. Wiley, New York (1999)

2. Bhushan, B.: Modern Tribology Handbook. CRC Press, Columbus (2001)

3. Zmitrowicz, A.: Wear Debris: a review of properties and constitutive models. J. Theor. Appl. Mech. 43(1), 3-35 (2005)

4. Soda, N.: Wear of some F.C.C. metals during unlubricated sliding. Part II: effects of normal load, sliding velocity and atmospheric pressure on wear fragments. Wear 35(2), 331-343 (1975)

5. MacQuarrie, R.A., Chen, Y.F., Coles, C., Anderson, G.I.: Wearparticle-induced osteoclast osteolysis: the role of particulates and mechanical strain. J. Biomed. Mater. Res. B Appl. Biomater. 69B, 104-112 (2004)

6. Green, T.R., Fisher, J., Stone, M., Wroblewski, B.M., Ingham, E.: Polyethylene particles of a "critical size" are necessary for the induction of cytokines by macrophages in vitro. Biomaterials $\mathbf{1 9}$, 2297-2302 (1998)

7. Elfick, A.P.D., Green, S.M., Krickler, S., Unsworth, A.: The nature and dissemination of UHMWPE wear Debris retrieved from periprosthetic tissue of THR. J. Biomed. Mater. Res. 65A, 95-108 (2003)

8. Hiratsuka, K., Muramoto, K.: Role of wear particles in severemild wear transition. Wear 259, 467-476 (2005)

9. Kato, H., Komai, K.: Tribofilm formation and mild wear by tribosintering of nanometer-sized oxide particles on rubbing steel surfaces. Wear 262(1-2), 36-41 (2006)

10. Hurley, S., Cann, P.M., Spikes, H.A.: Lubrication and reflow properties of thermally aged greases. Tribol. Trans. 43(2), 221-228 (2008)

11. Jin, X.: The effect of contamination particles on lithium grease deterioration. Lubr. Sci. 7(3), 233-245 (1995)

12. Olofsson, U., Olander, L., Jansson, A.: Towards a model for the number of airborne particles generated from a sliding contact. Wear 267, 2252-2256 (2009)

13. Vindedahlm, A., Strehlau, J.H., Arnold, W., Pen, R.L.: Organic matter and iron oxide nanoparticles: aggregation, interactions, and reactivity. Environ. Sci. Nano (6), 494-505 (2016)

14. Williams, J.A.: Wear and wear particles-some fundamentals. Tribol. Int. 38(10), 863-870 (2005)

15. Roylance, B.J., Williams, J.A., Dwyer-Joyce, R.: Wear Debris and associated phenomena-fundamental research and practice. Proc. Inst. Mech. Eng. Part J J. Eng. Tribol. 214(1), 79-105 (2000)

16. Kato, K.: Classification of wear mechanisms/models. J. Eng. Tribol. 216, 349-355 (2002)

17. Kato, K.: Micro-mechanisms of wear-wear modes. Wear 153(1), 277-295 (1992)

18. Mishina, H.: Surface deformation and formation of original element of wear particles in sliding friction. Wear 215(1-2), 10-17 (1998)
19. Mishina, H., Iwase, H., Hase, A.: Generation of wear elements and origin of tribomagnetization phenomenon. Wear 269(5-6), 491-497 (2010). doi:10.1016/j.wear.2010.05.004

20. Mishina, H., Hase, A.: Wear equation for adhesive wear established through elementary process of wear. Wear 308(1-2), 186-192 (2013). doi:10.1016/j.wear.2013.06.016

21. Hase, A., Mishina, H.: Wear elements generated in the elementary process of wear. Tribol. Int. 42(11-12), 1684-1690 (2008)

22. Scherge, M., Martin, J.M., Pohlmann, K.: Characterization of wear debris of systems operated under low wear-rate conditions. Wear 260, 458-461 (2006)

23. Wasche, R., Hartelt, M., Hodoroaba, V.-D.: Analysis of nanoscale wear particles from lubricated steel-steel contacts. Tribol. Lett. 58, 49-59 (2015)

24. Olofsson, U., Olander, L.: On the identification of wear modes and transitions using airborne wear particles. Tribol. Int. 59, 104-113 (2013)

25. Abbasi, S., Wahlstrom, J., Olander, L., Larsson, C., Olofsson, U., Sellgren, U.: A study of airborne wear particles generated from organic railway brake pads and brake discs. Wear 273, 93-99 (2011)

26. Weiner, B.: A guide to choosing a particle sizer. http://www. brookhaveninstruments.com/pdf/theory/AGuidetoChoosingaParticle. pdf

27. Pecora, R.: Dynamic Light Scattering: Application of Photon Correlation Spectroscopy. Plenum Press, New York (1985)

28. Skebo, J.E., Grabinski, A.M.S., Schlager, J.J., Hussain, S.M.: Assessment of metal nanoparticle agglomeration, uptake, and interaction using high-illuminating system. Int. J. Toxicol. 26, 135-141 (2007)

29. Zhou, D., Bennett, S.W., Keller, A.A.: Increased mobility of metal oxide nanoparticles due to photo and thermal induced disagglomeration. PLos one 7(5), 1-8 (2012)

30. Seipenbusch, M., Toneva, P., Peukert, W., Weber, A.P.: Impact fragmentation of metal nanoparticle agglomerates. Part. Part. Syst. Charact. 24, 193-200 (2007)

31. Kwak, K., Kim, C.: Viscosity and thermal conductivity of copper oxide nanofluid dispersed in ethylene glycol. Korea-Australia Rheol. J. 17(2), 35-40 (2005)

32. Völkl, A.: Ultracentrifugation. In: Encyclopedia of Life Sciences (ELS). John Wiley \& Sons, Ltd, Chichester (2010). doi:10.1002/ 9780470015902.a0002969.pub2

33. Rosická, D., Šembera, J.: Assessment of influence of magnetic forces on aggregation of zero-valent iron nanoparticles. Nanosc. Res. Lett. 6, 10-16(2011)

34. Chekli, L., Phuntsho, S., Roy, M., Lombi, E., Donner, E., Shon, H.K.: Assessing the aggregation behaviour of iron oxide nanoparticles under relevant environmental conditions using a multi-method approach. Water Res. 47, 4585-4599 (2013)

35. Merkus, H.G.: Particle size measurements. Fundamentals, practice, quality. Springer, Delft, Netherlands (2009)

36. Ordal, M.A., Bell, R.J., Alexander, R.W., Long, L.L., Querry, M.R.: Optical properties of fourteen metals in the infrared and far infrared: $\mathrm{Al} \mathrm{Co}, \mathrm{Cu}, \mathrm{Au}, \mathrm{Fe}, \mathrm{Pb}, \mathrm{Mo}, \mathrm{Ni}, \mathrm{Pd}, \mathrm{Pt}, \mathrm{Ag}, \mathrm{Ti}, \mathrm{V}$, and W. Appl. Opt. 24(24), 4493-4499 (1985)

37. Tallian, T.E.: The theory of partial elastohydrodynamic contacts. Wear 21, 49-101 (1972)

38. Dowson, D., Higginson, G.R.: New roller-bearing lubrication formula. Engineering (London) 192, 158-159 (1961)

39. Hooke, C.: A review of the paper 'A numerical solution to the elastohydrodynamic problem' by D. Dowson and G. R. Higginson. J. Eng. Tribol. 223, 49-53 (2009)

40. Spikes, H.A., Olver, A.V.: Basics of mixed lubrication. Lubr. Sci. 16, 3-28 (2003) 
41. Rounds, G.: Some environmental factors affecting surface coating formation with lubricating oil additives. ASLE Trans. 9, 88-106 (1966)

42. Quinn, T.F.J.: Review of oxidational wear part II: recent developments and future trends in oxidational wear research. Tribol. Int. 16(6), 305-315 (1983)

43. Ludema, K.: Friction, Wear, Lubrication: A Textbook in Tribology. CRC Press, Ann Arbor (1996)

44. Maccuspie, R.I., Rogers, K., Patra, M., Suo, Z., Allen, A.J., Martin, M.N.: Challenges for physical characterization of silver nanoparticles under pristine and environmentally relevant conditions. J. Environ. Monit. 13(5), 1212-1226 (2011)

45. Adamiak, M. (ed.): Abrasion Resistance of Materials. InTech (2012). doi:10.5772/1083

46. Padgurskas, J., Rukuiza, R., Prosyc, I., Kreivaitis, R.: Tribological properties of lubricant additives of $\mathrm{Fe}, \mathrm{Cu}$ and $\mathrm{Co}$ nanoparticles. Tribol. Int. 60, 224-232 (2013)
47. Chinas-Castillo, F., Spikes, H.A.: Mechanism of action of colloidal solid dispersions. J. Tribol. 125, 525-557 (2003)

48. Walker, D.: Chemical Reactions. Evans Brothers, London (2007)

49. Suh, N.P.: The delamination theory of wear. Wear 25, 111-124 (1973)

50. Bosman, R., Schipper, D.J.: Transition from mild to severe wear including running in effects. Wear 270(7-8), 472-478 (2011)

51. Jahanmir, S.: The relationship of tangential stress to wear particle formation mechanisms. Wear 103, 233-252 (1985)

52. Begelinger, A., de Gee, A.W.J.: Boundary lubrication of sliding concentrated steel contacts. Wear 22, 337-357 (1972)

53. Bosman, R., Hol, J., Schipper, D.J.: Running in of metallic surfaces in the boundary lubrication regime. Wear 271(7-8), 1134-1146 (2011) 